\title{
Performance of $\mathrm{Ar}^{+}$-Milled Ti:Sapphire Rib Waveguides as Single Transverse-Mode Broadband Fluorescence Sources
}

\author{
Christos Grivas, David P. Shepherd, Member, IEEE, Timothy C. May-Smith, Robert W. Eason, Markus Pollnau, \\ Aurelian Crunteanu, and Miroslav Jelinek
}

\begin{abstract}
Rib waveguides have been fabricated in pulsed-laser-deposited Ti:sapphire layers using photolithographic patterning and subsequent $\mathrm{Ar}^{+}$-beam milling. Fluorescence output powers up to $300 \mu \mathrm{W}$ have been observed from the ribs following excitation by a 3-W multiline argon laser. Mode intensity profiles show high optical confinement and the measured beam propagation factors $M_{x}^{2}$ and $M_{y}^{2}$ of 1.12 and 1.16, respectively, indicate single transverse-mode fluorescence emission. Loss measurements using the self-pumped phase conjugation technique have yielded comparable values $(1.7 \mathrm{~dB} / \mathrm{cm})$ for the ribs and the unstructured planar waveguide counterparts. The combination of optimum modal properties and strong optical confinement, together with sufficient levels of fluorescence output, make the single-moded Ti:sapphire rib waveguides a very interesting candidate as a fluorescence source for optical coherence tomography applications.
\end{abstract}

Index Terms-Ion beam applications, laser ablation, lithography, loss measurement, optical tomography, optical waveguides, rib waveguides.

\section{INTRODUCTION}

$\mathbf{O}$ PTICAL coherence tomography (OCT) is an interferometric imaging technique, which provides cross sectional images of diffusive media, such as biological tissue, with high longitudinal and transverse spatial resolutions. There are three general requirements for the light source used for OCT in biological imagining: 1) emission in the near infrared to ensure adequate penetration of the light in the tissue; 2) a large spectral bandwidth to ensure a short coherence length and, therefore, improved longitudinal resolution; and 3) high irradiance to achieve a wide dynamic range and detection sensitivity for imaging weakly backscattering structures in the tissue [1], [2]. Typical light sources for OCT for medical applications are edge light-emitting diodes (ELED) and superluminescent diodes (SLD), offering a longitudinal resolution of

Manuscript received September 24, 2002; revised November 13, 2002. The work of T. C. May-Smith was supported by the Engineering and Physical Sciences Research Council (EPSRC) under Grant GR/R74154.

C. Grivas, D. P. Shepherd, T. C. May-Smith, and R. W. Eason are with the Optoelectronics Research Centre, University of Southampton, Southampton SO17 1BJ, U.K. (e-mail: chg@orc.soton ac.uk).

M. Pollnau and A. Crunteanu are with the Applied Photonics Laboratory, Institute for Biomedical Imaging, Optics, and Engineering, Swiss Federal Institute of Technology, CH-1015 Lausanne, Switzerland.

M. Jelinek is with the Institute of Physics, Academy of Sciences of the Czech Republic, 18221 Prague 8, Czech Republic.

Digital Object Identifier 10.1109/JQE.2002.808149 $\sim 10-15$ and $15-30 \mu \mathrm{m}$, respectively, which is inadequate for subcellular imaging. Approximately $2-\mu \mathrm{m}$ resolution has been demonstrated using a Ti:sapphire bulk fluorescent emitter [3] and slow in vivo scanning can be performed in this way [4]. However, these sources are insufficient for fast-scanning OCT due to their low brightness. Recently, femtosecond Ti:sapphire lasers have been introduced as large-bandwidth high-brightness light sources offering a longitudinal resolution of $\sim 1 \mu \mathrm{m}$ [5].

In an attempt to develop light sources which combine high spectral power density, large bandwidth, and high spatial coherence, together with compactness and simplicity, we report here an investigation of the waveguiding characteristics of rib waveguide structures fabricated by $\mathrm{Ar}^{+}$-beam milling in Ti:sapphire films grown by pulsed laser deposition (PLD). The latter has been proven a reliable technique for the growth of waveguiding films of various laser host materials. Lasing has so far been observed in PLD grown $\mathrm{Nd}: \mathrm{Gd}_{3} \mathrm{Ga}_{5} \mathrm{O}_{12}$ [6], [7] and Ti:sapphire planar guides [8]. Recently, these Ti:sapphire waveguides have also demonstrated their suitability as broadband luminescence sources with a bandwidth that is applicable to the investigation of biotissue, and output powers sufficient to be considered for integration in existing OCT interferometric setups [9].

Rib waveguides show a number of advantages over their unstructured planar film counterparts such as lower threshold for laser structures, due to the lateral confinement of both the pump and laser modes, and improved efficiency provided that the rib fabrication process does not itself introduce any additional loss. Furthermore, their capability of providing a fully diffraction-limited, near-circular, single-mode beam makes them suitable for low loss coupling with components such as the fiber optic interferometers used in OCT systems. Fluorescence emission from a Ti:sapphire channel waveguide has previously been reported for ribs fabricated by reactive ion etching (RIE) [10]. Despite the multimode nature of the fluorescence output $\left(M_{x}^{2}=5.5-9\right.$ and $\left.M_{y}^{2}=2.3-2.9\right)$, its coupling efficiency to a single-mode fiber was already an order of magnitude higher than that from a planar guide.

This paper reports the substantial improvement in the modal properties and the fluorescence output for ribs patterned by $\mathrm{Ar}^{+}$-beam milling as characterized by the single-mode emission and the almost five-times higher output powers than the previously reported RIE-fabricated counterparts [10]. Additionally, we have assessed the fabrication technique in terms of its potential to have minimum contribution to background 
(a)

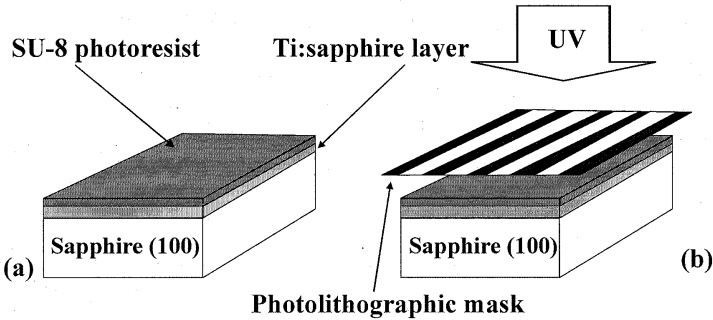

(c)

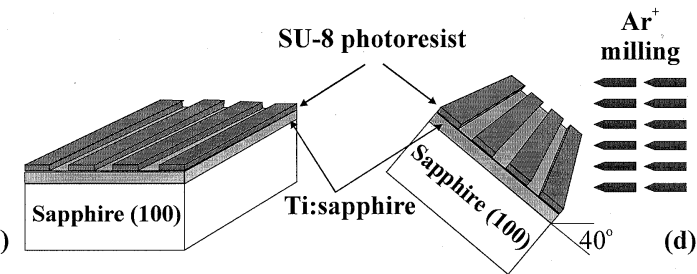

(e)

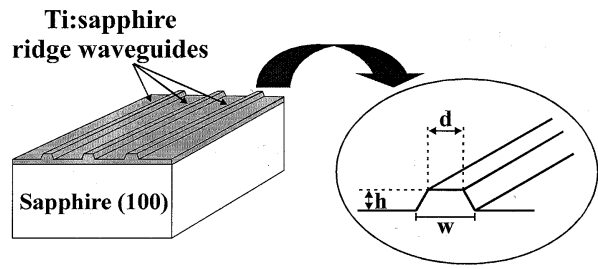

Fig. 1. Schematic of the rib fabrication steps in Ti:sapphire films. (a) Application of a negative photoresist on top of a Ti:sapphire layer by spin coating. (b) Exposure to UV light (mask aligner). (c) Development to produce stripped patterns of photoresist with a rib shape. (d) Ion beam milling to etch the uncovered Ti:sapphire surfaces. (e) Removal of the photoresist remnants to obtain rib structures. The inset shows details of the resulting structures.

propagation loss in the host film by performing loss measurements using the self-pumped phase conjugation (SPPC) technique [11]. Finally, we have investigated the possibility to obtain higher fluorescence output using amplified spontaneous emission as well as laser action.

\section{EXPERIMENTAL DETAILS}

\section{A. Fabrication Techniques}

Depositions were performed in a stainless steel vacuum chamber, which was evacuated down to a base pressure of $5 \times 10^{-6}$ mbar. Films were grown by pulsed laser ablation of a single crystal Ti:sapphire target of $0.12 \mathrm{wt} \% \mathrm{Ti}_{2} \mathrm{O}_{3}$ in a background argon atmosphere of $3 \times 10^{-4}$ mbar. Ablation was provided by a $\mathrm{KrF}$ excimer laser (Lambda Physik, LPX 200, $248 \mathrm{~nm}$, pulse duration $\sim 20 \mathrm{~ns}$ ) operated at $25 \mathrm{~Hz}$, and focused to an energy density of $\sim 4 \mathrm{~J} / \mathrm{cm}^{2}$ on the target. Films were deposited on sapphire (100) substrates positioned at a distance of $4 \mathrm{~cm}$ away from the target material. All films employed for the investigation were grown at substrate temperatures of $\sim 975^{\circ} \mathrm{C}$ and had a thickness of $\sim 11 \mu \mathrm{m}$. To localize the substrate heating and to prevent contamination through desorption from the walls of the deposition chamber, a $100 \mathrm{~W} \mathrm{CO}_{2}$ laser (Synrad 57-1-28 W) was used as a heating source. This heating technique has already been implemented successfully in PLD [12], [13].

The rib waveguide fabrication is illustrated in Fig. 1. In the first step, a $10-\mu$ m-thick layer of negative photoresist (SU-8,

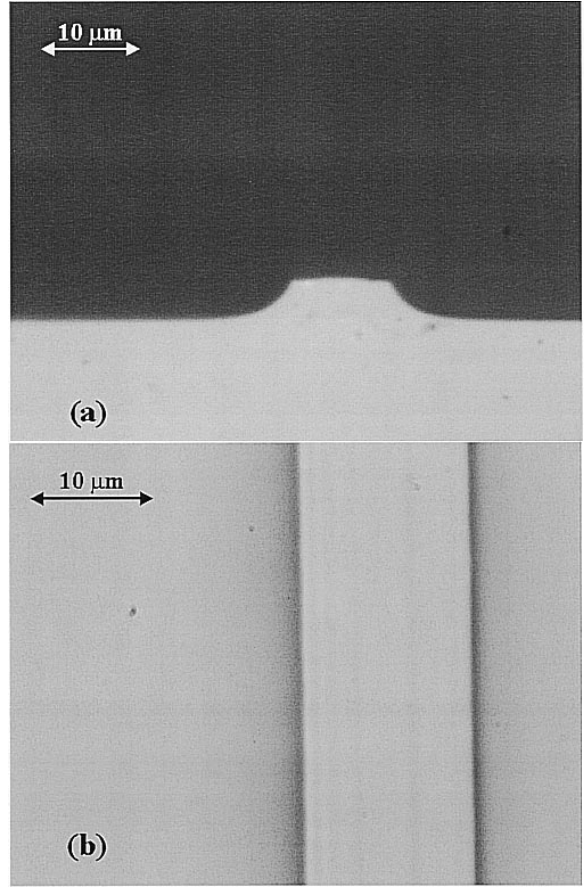

Fig. 2. Optical microscope picture of: (a) the polished end-face of a Ti:sapphire rib waveguide and (b) top view of the same structure.

Microchem) was applied on the top of the PLD-grown Ti:sapphire waveguide [Fig. 1(a)] and then was photolithography patterned using a chromium mask and irradiation from a UV lamp [Fig. 1(b) and (c)]. Prior to the coating procedure, the sample had been dehydrated by maintaining it at a temperature of $80^{\circ} \mathrm{C}$ for $10 \mathrm{~min}$. Subsequently, the sample was mounted on a rotating holder and $\mathrm{Ar}^{+}$-beam milling was used [Fig. 1(d)] to etch the exposed parts of the waveguide to produce an array of $5-\mu \mathrm{m}$ deep Ti:sapphire ribs with widths $w$ varying from 6 to $16 \mu \mathrm{m}$ separated by $100 \mu \mathrm{m}$ [Fig. 1(e)]. As shown in the inset in Fig. 1, the ribs had a trapezoidal profile. The width at the top of the structure $d$ was $60 \%$ of the corresponding value $w$ at the base for the $w=14 \mu \mathrm{m}$ rib, which is also used for our simulations in Section II-B. The etch rate of the Ti:sapphire and the SU-8 photoresist using a 500- $\mathrm{V}$ neutralized $\mathrm{Ar}^{+}$beam with an ion density of $0.9 \mathrm{~mA} / \mathrm{cm}^{2}$ in a background pressure $2 \times 10^{-6} \mathrm{mbar}$ and at an exposure angle of $40^{\circ}$ were 13 and $24 \mathrm{~nm} / \mathrm{min}$ respectively. The exposure angle in the milling process has an impact on the width ratio $(d / w)$ in a way that higher angles lead to more inclined side faces. Following $\mathrm{Ar}^{+}$milling, all the photoresist remnants were completely removed from the top of the guide by ultrasonic cleaning in an acetone bath. After all the fabrication steps had been completed, the end faces of the sample were polished to an optical finish to optimize the in- and out-coupling procedures. Fig. 2 shows optical microscope pictures of a typical profile and a top view of one of the resulting guides.

\section{B. Loss Measurements and Transmission Characteristics}

Nondestructive propagation loss measurements for the 5.2-mm-long rib waveguides were performed via the SPPC method [11] using the experimental arrangement shown in Fig. 3. The output from a Ti:sapphire laser (Spectra Physics, Model 3900S) operating at $720 \mathrm{~nm}$ was launched into the 


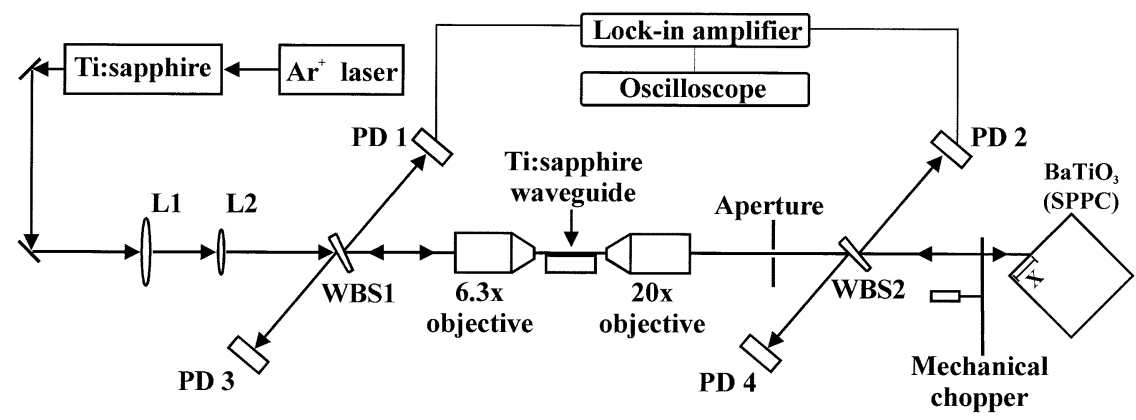

Fig. 3. Experimental arrangement for loss measurements via self-pumped phase conjugation.

rib waveguides and then coupled out using two microscope objectives with a magnification of $\times 6.3$ and $\times 20$, respectively. This short Ti:sapphire wavelength was chosen so that the combination of output power and operating wavelength provided optimal crystal response in terms of intensity and build-up time of the phase conjugated signal. To optimize the coupling efficiency for the Ti:sapphire laser, the beam was shaped to an aspect ratio of 2.5:1 to adequately fit that of the modes of the guide using a cylindrical lens telescope, (L1 and L2, focal lengths $f=25 \mathrm{~cm}$ and $10 \mathrm{~cm}$ respectively). The outcoupled waveguide mode was phase conjugated via SPPC from a nominally undoped $\mathrm{BaTiO}_{3}$ crystal and the retro-reflected beam was thereby automatically coupled back into the waveguide without any launch losses other than the Fresnel reflections. Using the standard total internal reflection (TIR) geometry [14] the $\mathrm{BaTiO}_{3}$ crystal was mounted on a rotation-translation stage so that the crystal response could be optimized by varying the transverse position of the beam input and the angle of incidence. An aperture was positioned at the exit face of the sample to ensure that only the rib waveguide mode underwent phase conjugate reflection. Two $1^{\circ}$ wedged beam splitters (WBS1, 2) and two photodiodes (PD1, 2) connected to a lock-in amplifier respectively were used to monitor the incident as well as the phase-conjugate signal before and after its passing through the rib waveguides. Modulation for the signal, which was fed to the lock-in amplifier, was provided by a mechanical chopper positioned between the wedged beam splitter (WBS2) and the crystal. With this configuration, the SPPC signal was distinguished from all other reflections resulting from the optical elements and more importantly the waveguide faces. The power of the incident beam at the $\mathrm{BaTiO}_{3}$ crystal input face was $11.4 \mathrm{~mW}$ and had a diameter of $\sim 1.5 \mathrm{~mm}$ as it entered the crystal. The maximum steady-state reflectivity was found to be $47 \%$ of the input intensity at an incident angle of $42^{\circ}$ with respect to the normal to the crystal surface and at a transverse position of $x=2 \mathrm{~mm}$ on the $5.7-\mathrm{mm}$-wide crystal face. The build-up time of the reflected signal was $\sim 3 \mathrm{~min}$. The waveguide loss in decibels per unit length $\alpha$ was therefore derived from the following equation:

$$
\alpha=\left(\frac{1}{L}\right) \cdot 10 \cdot \log _{10}\left[\left(\frac{1}{K}\right) \cdot\left(\frac{P D 1}{P D 2}\right)\right]
$$

where $L$ is the length of the rib waveguide and $K$ is the transmission factor which includes all power losses due to the op- tical elements and the Fresnel reflection from the coupling and decoupling faces of the waveguide. It is given by

$$
K=\left(\frac{T_{B S 2}}{R_{B S 2}}\right) \cdot\left(R_{B S 1}\right) \cdot\left(T_{O 1}\right) \cdot\left(T_{O 2}\right) \cdot\left(T_{\mathrm{FR}}\right)^{2}
$$

where $R_{B S 1}$ and $R_{B S 2}$ are the reflection coefficients for the wedged beam splitters WSB1 and WSB2, respectively, $T_{B S 2}$ stands for the transmission coefficient for the beam splitter WSB2, $T_{O 1}$ and $T_{O 2}$ represent the transmission coefficients for the coupling and decoupling objective respectively, and $T_{\mathrm{FR}}$ is the Fresnel transmission coefficient at the waveguide-air interface. $T_{\mathrm{FR}}$ is given by the equation

$$
T_{\mathrm{FR}}=1-\left[\frac{(1-n)}{(1+n)}\right]^{2}
$$

where $n$ is the refractive index of the waveguiding material. For Ti:sapphire ( $n=1.76$ ), the Fresnel transmission coefficient is 0.93. After having determined $K$ by measuring the terms in (2), we measured the ratio PD1/PD2 as described above, and thereby evaluated the waveguide loss from (1). The propagation loss for the rib waveguide was $1.7 \pm 0.1 \mathrm{~dB} / \mathrm{cm}$, with reproducibility within an error of $5 \%$ for a set of five measurements. Comparison between this value and the one obtained for the Ti:sapphire planar waveguide host [8] leads to the conclusion that the loss level remains essentially unchanged after the rib fabrication process. We further note that no dependence of the loss on the size of the ribs was observed over the range of values between 9 and $16 \mu \mathrm{m}$. Ribs with widths smaller than $9 \mu \mathrm{m}$ have shown higher losses ( $\geq 5 \mathrm{~dB} / \mathrm{cm}$ ) due to imperfections in the fabrication process arising from the photolithographic patterning.

The throughput of the Ti:sapphire laser beam from a 5 - $\mu \mathrm{m}$-deep rib waveguide with a width $w$ of $14 \mu \mathrm{m}$, which was on top of a 5- $\mu \mathrm{m}$-thick Ti:sapphire film, was imaged onto a CCD camera. The experimentally recorded profile, shown in [Fig. 4(a)] along with the calculated mode profile at $800 \mathrm{~nm}$ [Fig. 4(b)], was Gaussian in the horizontal and nearly Gaussian in the vertical plane of the guide. The asymmetry in the vertical direction is due to the asymmetric refractive index profile in this axis. From the size of the image on the CCD camera, a spot size of $3.6 \times 5.8 \mu \mathrm{m}^{2}$ has been calculated for the guided mode, which is in good agreement with the simulated fundamental mode intensity profile shown in Fig. 4(b). The simulation, which was performed using a software package from Kymata Software using the finite-difference method, suggests that the guide supports only one mode and this also agrees with the 


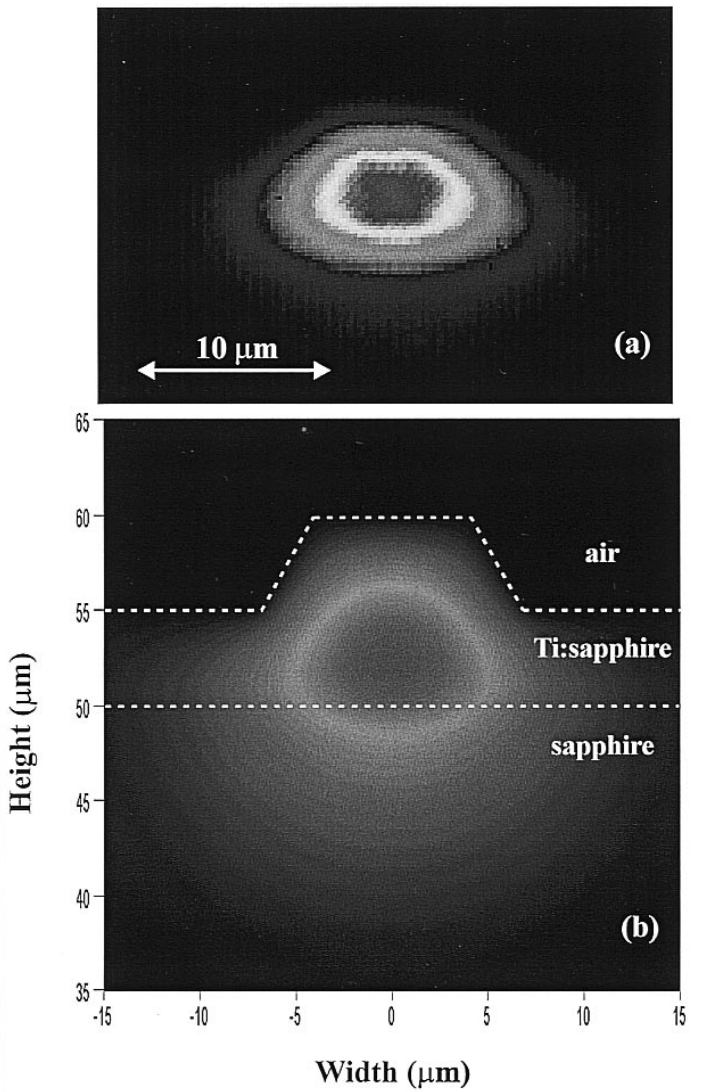

Fig. 4. (a) Mode intensity profiles of the out-coupled light for 100-mW in-coupled power from a Ti:sapphire laser operating at $800 \mathrm{~nm}$. The $5-\mu \mathrm{m}$-deep rib structure had a width $w$ of $14 \mu \mathrm{m}$ and was on top of a $5-\mu \mathrm{m}$-thick Ti:sapphire film. (b) Simulated fundamental mode intensity profile for a Ti:sapphire rib structure with the same dimensions.

experimental observation. We have assumed an increase in the refractive index $\Delta n=3.7 \times 10^{-4}$ for the Ti:sapphire film compared to the sapphire substrate, which corresponds to a $\mathrm{Ti}$ concentration of $0.1-\mathrm{wt} \%$. This value of doping level was inferred by taking into account that the Ti:sapphire crystal target used for the PLD experiments had a concentration of 0.12 -wt $\%$ in $\mathrm{Ti}_{2} \mathrm{O}_{3}$, and is entirely consistent with the results from the Particle-Induced X-ray Excitation (PIXE) investigation [8], which has shown that $\sim 80 \%$ of the $\mathrm{Ti}$ can be transferred from the target to the PLD layer under the growth conditions described in Section II-A. The single transverse-mode nature of the outcoupled beam was also confirmed by measuring the beam propagation factors $\left(M^{2}\right)$ with a Coherent Modemaster beam propagation analyzer, with values of 1.03 and 1.09 , respectively, for the parallel and the perpendicular directions with respect to the waveguide plane. It should be noted that this does not prove that the guide only supports one transverse mode, as single-mode propagation in a multimode guide is possible. However, it is certainly consistent with a single-mode guide. A stronger test of the single-mode nature of the guide will be given by looking at the fluorescent output, as this will be coupled to all the supported modes.

\section{Fluorescence Characteristics}

The fluorescence characteristics were examined with an argon laser as the pump source (Coherent, Model Innova 70)
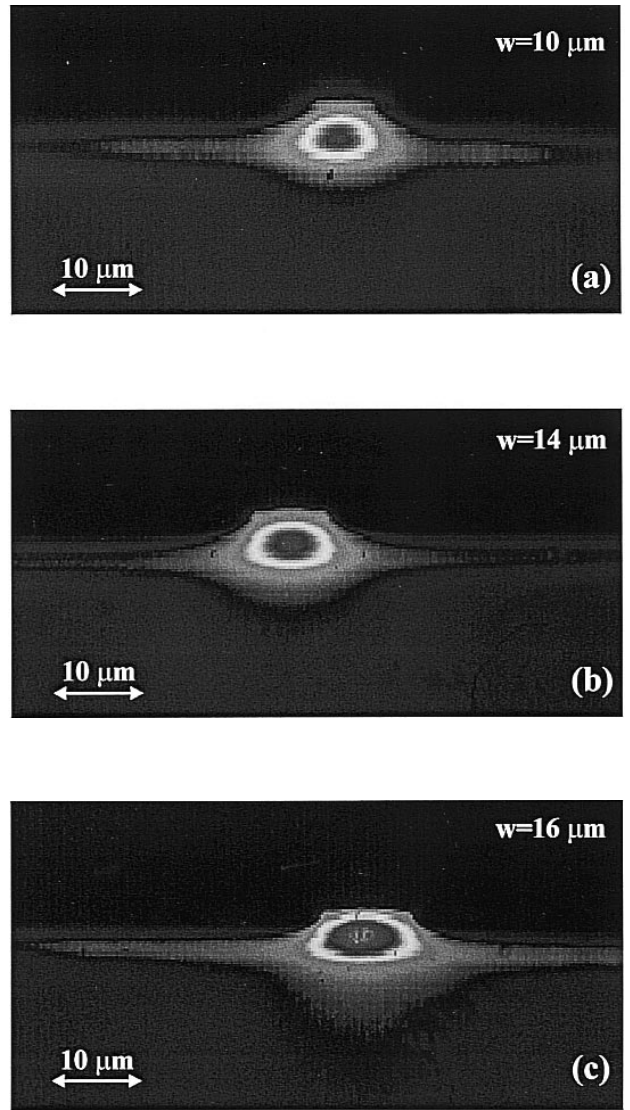

Fig. 5. Fluorescence emission profiles measured at the exit face of a Ti:sapphire rib waveguide with a depth of $5 \mu \mathrm{m}$ and widths $w$ of: (a) 10, (b) 14, and (c) $16 \mu \mathrm{m}$. The thickness of the Ti:sapphire underlying layer was $5 \mu \mathrm{m}$.

operating on all lines. The fluorescence emission, which was collected from the exit face of the guide, was imaged on the CCD camera after having passed through an OG 550 filter to block the residual transmitted pump irradiation. It was then masked by an aperture to ensure that only the fluorescence from the rib guide itself and not from the planar region between the ribs, was measured. The experimentally recorded intensity profiles of the fluorescence (without the aperture) originating from ribs with a height of $5 \mu \mathrm{m}$ and widths $w$ varying from 10 to $16 \mu \mathrm{m}$ are shown in [Fig. 5]. The profiles indicate that the guides are suitable for producing strong optical confinement. Measurements of the beam propagation factors show diffraction limited output with values of 1.12 and 1.16 , respectively, for the parallel and the perpendicular directions with respect to the waveguide plane. This is essential to ensure efficient coupling to the fiber-optic interferometric arrangements used in OCT systems. The strong optical confinement of the fluorescence emission also indicates the potential for fabrication of closely spaced (in the order of a few tens of micrometers) multiple ribs on the same substrates.

Fig. 6 shows the fluorescence output power from waveguides with different widths as a function of the input power. The maximum output powers were $283 \mu \mathrm{W}(w=16 \mu \mathrm{m}), 243 \mu \mathrm{W}$ $(w=14 \mu \mathrm{m})$ and $181 \mu \mathrm{W}(w=10 \mu \mathrm{m})$, and the corresponding slope efficiencies were $9.5 \times 10^{-5}, 8.1 \times 10^{-5}$, and $6.2 \times 10^{-5}$.

To make a rough prediction of the theoretical fluorescence power as a function of the pumping power and the rib size, we 


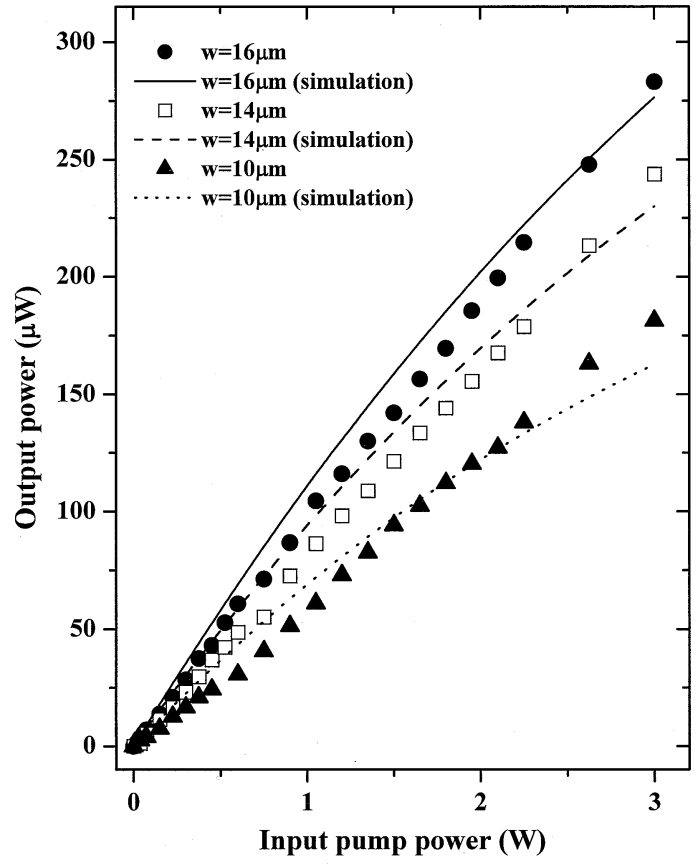

Fig. 6. Fluorescence power as a function of pump power from an $\mathrm{Ar}^{+}$laser for 5- $\mu$ m-deep ribs with a width at the bottom of: $(\bullet) 16$, ( $\square) 14$, and $(\mathbf{\Delta}) 10 \mu \mathrm{m}$. The full line refers to the calculated fluorescence output for a rib with a width of $16 \mu \mathrm{m}$, the dashed line to $14 \mu \mathrm{m}$, and the dotted one to $10 \mu \mathrm{m}$. For the calculations, we assume 5 - $\mu \mathrm{m}$-deep ribs.

follow a plane-wave analysis accounting for depletion of the absorbing level as outlined in [15]. We assume a four-level system with population in either the emitting level or in the ground state. The population density $n_{2}$ in the emitting level will vary along the propagation direction $y$ of the guide, and so we define a length integrated population density given by

$$
N_{2}=\int_{0}^{L} n_{2}(y)
$$

where $L$ is the length of the waveguide. The fraction of input pump power that is absorbed in the waveguide is

$$
F_{A}=1-e^{\sigma_{p}\left(N_{2}-n_{o} L\right)}
$$

where $\sigma_{p}=6 \times 10^{-20} \mathrm{~cm}^{2}$ is the absorption cross section for $\pi$ polarization at $515 \mathrm{~nm}[19]$, and $n_{0}=2.6 \times 10^{-19} / \mathrm{cm}^{3}$ corresponds to the total dopant density of Ti and was obtained by assuming that the sample had a doping level equivalent to $0.1-w t \%$ Ti. The excitation rate induced by the pump beam is then given by the expression

$$
R_{\mathrm{ex}}=\frac{P_{p} \cdot \eta_{\mathrm{del}}}{h \cdot v_{p}} \cdot\left[1-e^{\sigma_{p}\left(N_{2}-n_{o} L\right)}\right]
$$

where $P_{p}$ is the pump laser input power, $h$ is Planck's constant, $v_{p}$ is the frequency of the pump irradiation, and $\eta_{\text {del }}$ is the delivery efficiency of the pump into the guide and accounts for the launch efficiency as well as for losses originating from the optics delivery system to the guide. The parameter $\eta_{\text {del }}$ is strongly dependent on the size of the guides and the measured values for ribs with a width $w$ of 10,14 , and $16 \mu \mathrm{m}$ were, correspondingly,
$0.46,0.6$, and 0.69 for our optical setup. The rate of de-excitation due to the spontaneous emission is:

$$
R_{\text {de-ex }}=\frac{N_{2} \cdot A}{\tau}
$$

where $A$ is the cross-sectional area of the pumped region and $\tau=3.2 \mu \mathrm{s}$ [16] is the excited-state lifetime (we neglect nonradiative lifetime quenching with increasing temperature owing to sample heating under laser pumping). Assuming steady-state conditions, we set the rate of excitation of the $\mathrm{Ti}^{3+}$ ions in (6) equal to de-excitation rate in (7). The resulting expression allows us to calculate $N_{2}$ and subsequently the total fluorescence power $P_{\text {tot }}$ as a function of input power. The latter is deduced from

$$
P_{\mathrm{tot}}=N_{2} \cdot \frac{A \cdot h \cdot v_{l}}{\tau}
$$

where $v_{l}$ is the frequency of the fluorescence output, which we take as the value at the centre of the fluorescence spectrum at $v_{l}=3.75 \times 10^{14} \mathrm{~Hz}$. The fraction of the total fluorescence power that is captured by the numerical aperture (NA) of the waveguide, and therefore provides useful guided output $P_{\mathrm{f}}$, is given by [17]

$$
P_{\mathrm{fl}}=P_{\mathrm{tot}} \cdot \frac{\Omega}{4 \cdot \pi}
$$

where $\Omega$ is the solid angle captured by the waveguide NA and is given by

$$
\Omega=\frac{\pi \cdot \theta^{2}}{n^{2}}
$$

where $n$ is the refractive index of the waveguide and $\theta$ is the divergence of the waveguide output and is related to the NA by

$$
N A=\sin \theta \cong \theta \text {. }
$$

As an estimate of $\theta$, and hence the effective NA of our rib guides, we take the divergence of the smallest mode-size obtained $(77 \mathrm{mrad})$. The lines in Fig. 6 represent the fluorescence power as calculated from (9) as a function of input pump power for rib structures of various widths $w$. We observe that fluorescence output levels for ribs with different size derived by our basic model calculations are in agreement with the experimental results. The curvature of the theoretical lines, which is more evident for the smaller in size ribs, is due to saturation of the absorption in the guide, although this effect was not clearly observed experimentally over the investigated pump energy range.

Measurements of the fluorescence power have also been performed with a mirror of high reflectivity at the emission wavelength range of the guide and a transmission of $86 \%$ for the pump beam butted to the incoupling face of the guide. This configuration leads to an increase of $\sim 46 \%$ in the emitted fluorescence power, which results from the back reflection of the counter propagating luminescence. Thus, as shown in Fig. 7, for a rib with a width $w$ of $14 \mu \mathrm{m}$, the maximum output power increases from 243 to $350 \mu \mathrm{W}$, and the corresponding slope efficiencies from $8.1 \times 10^{-5}$ to $1.2 \times 10^{-4}$. 


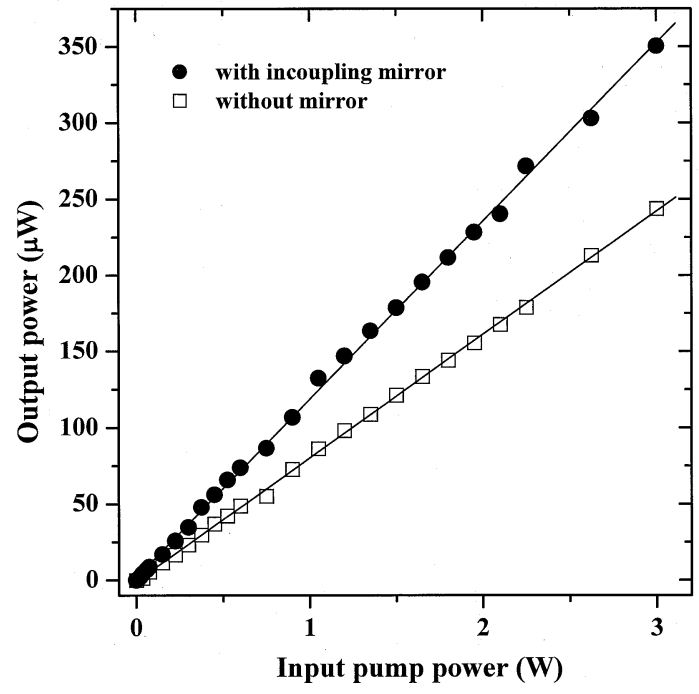

Fig. 7. Fluorescence output as a function of pump power from an $\mathrm{Ar}^{+}$laser for 5- $\mu \mathrm{m}$-deep ribs with a width at the bottom of $14 \mu \mathrm{m}$ with $(\bullet)$ and without ( $\square$ ) incoupling mirror.

\section{CONCLUSIONS}

In summary, rib waveguides based on PLD-grown Ti:sapphire thin films have been fabricated by $\mathrm{Ar}^{+}$milling. Mode-intensity profiles and fluorescence output power measurements have shown that the specific waveguide geometry leads to a strong optical confinement and maintains the fluorescence output efficiency at reasonable levels compared to their thin-film counterparts. Furthermore, nondestructive loss measurements using the self-pumped phase conjugation technique have shown that the additional fabrication steps required to obtain the rib waveguides do not significantly increase the value of propagation loss compared to the background loss level of other PLD-grown Ti:sapphire thin films which were between 1.6 and $1.8 \mathrm{~dB} / \mathrm{cm}$. The optimum modal properties, high fluorescence output, and its compactness make it a suitable fluorescence source for integration with fiber-optic OCT systems. Future work will concentrate on decreasing the background losses in the PLD-grown Ti:sapphire host film waveguides and as a consequence those in the rib structures, as well as on investigating the use of amplified spontaneous emission to increase the output power without too large a decrease in bandwidth. It is also interesting to note that, even with the current level of propagation losses, demonstration of laser action in a Ti:sapphire channel waveguide should be possible [8].

\section{ACKNOWLEDGMENT}

The authors are grateful to $\mathrm{M}$. Damzen from Imperial College, London for the loan of a $\mathrm{BaTiO}_{3}$ crystal.

\section{REFERENCES}

[1] D. Huang, E. A. Swanson, C. P. Lin, J. S. Schuman, W. G. Stinson, W. Chang, M. R. Hee, T. Flotte, K. Gregory, C. A. Puliafito, and J. G. Fujimoto, "Optical coherence tomography," Science, vol. 254, pp. 1178-1181, 1991.

[2] J. M. Schmitt, "Optical coherence tomography (OCT): A review," IEEE J. Select. Topics Quantum Electron., vol. 5, pp. 1205-1215, 1999.

[3] X. Clivaz, F. Marquis-Weible, and R. P. Salathé, "Optical low coherence reflectometry with $1.9 \mu \mathrm{m}$ spatial-resolution," Electron. Lett., vol. 28, p. 1553, 1992.

[4] A. Kowalevicz, T. Ko, I. Hartl, J. G. Fujimoto, M. Pollnau, and R. P. Salathé, "Ultrahigh resolution optical coherence tomography using a superluminescent light source," Opt. Exp., vol. 10, pp. 349-353, 2002.

[5] W. Drexler, U. Morgner, F. X. Kärtner, C. Pitris, S. A. Boppart, X. D. Li, E. P. Ippen, and J. G. Fujimoto, "In vivo ultrahigh-resolution optical coherence tomography," Opt. Lett., vol. 24, pp. 1221-1223, 1999.

[6] D. S. Gill, A. A. Anderson, R. W. Eason, T. J. Warburton, and D. P. Shepherd, "Laser operation of an Nd: $\mathrm{Gd}_{3} \mathrm{Ga}_{5} \mathrm{O}_{12}$ thin-film optical waveguide fabricated by pulsed laser deposition," Appl. Phys. Lett., vol. 69, pp. 10-12, 1996.

[7] C. L. Bonner, A. A. Anderson, R. W. Eason, D. P. Shepard, D. S. Gill, C. Grivas, and N. A. Vainos, "Performance of a low-loss pulsed-laserdeposited $\mathrm{Nd}: \mathrm{Gd}_{3} \mathrm{Ga}_{5} \mathrm{O}_{12}$ waveguide laser at 1.06 and $0.94 \mu \mathrm{m}$," Opt. Lett., vol. 22, pp. 988-990, 1997.

[8] A. A. Anderson, R. W. Eason, L. M. B. Hickey, M. Jelinek, D. S. Gill, C. Grivas, and N. A. Vainos, "Ti:sapphire planar waveguide laser grown by pulsed laser deposition," Opt. Lett., vol. 22, pp. 1556-1558, 1997.

[9] M. Pollnau, R. P. Salathé, T. Bhutta, D. P. Shepherd, and R. W. Eason, "Continuous-wave broadband emitter based on transition-metal-ion-doped waveguide," Opt. Lett., vol. 26, pp. 283-285, 2001.

[10] A. Crunteanu, M. Pollnau, G. Jänchen, C. Hibert, P. Hoffman, R. P. Salathé, R. W. Eason, C. Grivas, and D. P. Shepherd, "Ti:sapphire rib channel waveguide fabricated by reactive ion etching of a planar waveguide," Appl. Phys. B, vol. 75, pp. 15-17, 2002.

[11] S. Brülisauer, D. Fluck, C. Solcia, T. Pliska, and P. Günter, "Nondestructive waveguide loss-measurement method using self-pumped phase conjugation for optimum end-fire coupling," Opt. Lett., vol. 20, pp. 1773-1775, 1995.

[12] S. J. Barrington and R. W. Eason, "Homogeneous substrate heating using $\mathrm{a} \mathrm{CO}_{2}$ laser with feedback, rastering, and temperature monitoring," Rev. Sci. Instrum., vol. 71, pp. 4223-4225, 2000.

[13] A. A. Anderson, R. W. Eason, M. Jelinek, C. Grivas, D. Lane, K. Rodgers, L. M. B. Hickey, and C. Fotakis, "Growth of Ti:sapphire single crystal thin films by pulsed laser deposition," Thin Solid Films, vol. 300, p. 68, 1997.

[14] J. Feinberg, "Self-pumped, continuous-wave phase conjugator using internal-reflection," Opt. Lett., vol. 7, pp. 486-488, 1982.

[15] R. J. Beach, "CW theory of quasithree level end-pumped oscillators," Opt. Commun., vol. 123, p. 385, 1995.

[16] P. F. Moulton, "Spectroscopic and laser characteristics of $\mathrm{Ti}^{-\mathrm{Al}_{2} \mathrm{O}_{3}}$," $J$. Opt. Soc. Amer. B, vol. 3, pp. 125-133, 1986.

[17] E. Desurvire, Erbium-Doped Fiber Amplifiers. New York: Wiley, 1994, ch. 1.

Christos Grivas received the diploma degree in physics from the University of Crete, Crete, Greece, and the Ph.D. degree in physics from the Johannes Kepler University, Linz, Austria.

From 1994 to 1997, he was with the Foundation for Research and Technology-Hellas (FO.R.T.H.), Heraklion, Greece. In 1998, he joined the National Institute of Materials and Chemical Research, Tsukuba, Japan, to conduct research as a part of a European Union STF fellowship and then as part of a Japanese Science and Technology Agency Centre of Excellence Programme. In 2001, he joined the Optoelectronics Research Centre, Southampton, U.K. His research activities are in the areas of laser material processing and film-deposition techniques, materials development for optoelectronic applications, organic photochemistry, and chemical physics. He has authored over 25 papers in refereed journals. 
David P. Shepherd (M'01) received the B.Sc. degree in physics in 1985 and the Ph.D. degree in laser physics in 1989 from the University of Southampton, Southampton, U.K. His thesis topic was the development of short-pulse sources at $1.5 \mu \mathrm{m}$ and involved research in Raman scattering and $\mathrm{Yb}$ :Er:Glass bulk and fiber lasers.

He was then a Research Fellow in the Physics Department and, as of 1991, in the Optoelectronics Research Centre at the University of Southampton, investigating planar waveguide lasers based on rare-earth-ion and transition-metal-ion doped crystals and glasses, including work on waveguides fabricated by ion-implantation, ion-diffusion, ion-exchange, liquid-phase-epitaxy, molecular-beam epitaxy, pulsed laser deposition, spin coating and direct bonding. He is currently a Principal Research Fellow, leading a group investigating waveguide devices primarily for high-power diode-pumped laser sources. His research interests also include self-adaptive gain-grating lasers and synchronously-pumped optical parametric oscillators. He has published over 60 papers in scientific journals, mostly concerned with planar waveguide lasers.

Timothy C. May-Smith was born in Trowbridge, Wiltshire, U.K., in 1978. He received the M.Phys. degree from the University of Wales, Swansea, U.K., in 2001. He is currently working toward the Ph.D. degree at the Optoelectronics Research Centre, University of Southampton, Southampton, U.K., where he is studying planar waveguides fabricated using the pulsed laser deposition technique.

His current lines of research include the fabrication of multilayer garnet crystal waveguides with rare-earth-ion doped cores for high-powe diode-pumped lasers and titanium-doped sapphire waveguides for broadband luminescence applications and ultra-short pulse lasers.

Robert W. Eason received the undergraduate and postgraduate degrees from University College London, London, U.K., in 1975, and from York University, York, U.K., in 1981.

$\mathrm{He}$ is a Professor of Optoelectronics in the Optoelectronics Research Centre and Department of Physics and Astronomy, University of Southampton, Southampton, U.K. He has also worked at Bristol University, the Rutherford Appleton Laboratory, and Essex University (as a new-blood lecturer). $\mathrm{He}$ lectures in optics and laser science, and heads a research group investigating a range of nonlinear and applied optical phenomena including phase-conjugate laser resonators, photorefraction, optical waveguides, pulsed laser deposition, bonding and etching techniques, microstructuring of nonlinear materials, and ferroelectric domain engineering for application in opto-electro-micromechanical systems. He has served on numerous national and international committees, is a current member of the Engineering and Physical Sciences Research Council (EPSRC) physics college.
Markus Pollnau received the Diploma in Physics from the University of Hamburg, Hamburg, Germany, in 1992 and the Ph.D. degree in physics from the University of Bern, Bern, Switzerland, in 1996.

From 1996 to 1998, he worked at the University of Southampton, Southampton, U.K., as Research Fellow of the European Union, and from 1998-1999 at the University of Bern. From 1998 to 2000, he was also a Visiting Researcher at the University of Manchester, Manchester, U.K. In 1999, he joined the Institute of Imaging and Applied Optics, Swiss Federal Institute of Technology, Lausanne, Switzerland, as a "Profil" fellow of the Swiss National Science Foundation. His current research interests are in the fields of broadband light sources based on transition-metal-ion and rare-earth-ion doped waveguide structures for applications in optical coherence tomography and mid-infrared fiber lasers for applications in micro-surgery. He has co-authored over 100 technical contributions in international journals and conferences.

Dr. Pollnau is a member of the European, German, and Swiss Physical Societies and the Optical Society of America (OSA).

Aurelian Crunteanu received the Phys.Eng. degree in optics and optical technologies in 1995 and the Master's degree in 1996, both from the University of Bucharest, Bucharest, Romania. He received the Ph.D. degree in physics from the University of Bucharest in 2000 and the Ph.D. degree in material sciences from the Université Claude Bernard-Lyon1, France, in 2001, in the framework of a co-directed doctoral thesis.

He was also a Research Assistant at the National Institute for Lasers, Plasma and Radiation Physics, Bucharest, Romania, from 1995 to 2000. In 2001, he obtained a post-doctoral fellowship at the Institute of Imaging and Applied Optics at the Swiss Federal Institute of Technology, Lausanne. His current research interests focus on the field of microtechnology, including the fabrication and characterization of micro- and nano-structures in laser-host materials, the optical investigation of planar and channel waveguides, and laser-assisted thin-film deposition. He is the co-author of more than 35 contributions in international journals and conferences.

Miroslav Jelinek was born in 1946. He received the Ph.D. degree in 1978 and the Dr. Sci. degree in 1999, both from the Czech Technical University, Prague, Czechoslovakia.

Since 1984, he has been with Institute of Physics, Academy of Sciences of the Czech Republic, Prague. His current research interest is in laser ablation, including deposition, thin films, and laser applications. He has more than 80 publications in these areas.

Dr. Jelinek was the recipient of the SPIE Best Paper Award in 1996. 